\title{
Valproic Acid Limits Pancreatic Recovery after Pancreatitis by Inhibiting Histone Deacetylases and Preventing Acinar Redifferentiation Programs
}

John F. Eisses, ${ }^{*}$ Angela Criscimanna, ${ }^{\dagger}$ Zachary R. Dionise, ${ }^{*}$ Abrahim I. Orabi, ${ }^{*}$ Tanveer A. Javed, ${ }^{*}$ Sheharyar Sarwar, ${ }^{*}$ Shunqian Jin, ${ }^{*}$ Lili Zhou, ${ }^{\ddagger}$ Sucha Singh, ${ }^{\ddagger}$ Minakshi Poddar, ${ }^{\ddagger}$ Amy W. Davis, ${ }^{\S}$ Akif Burak Tosun, ${ }^{\natural}$ John A. Ozolek, ${ }^{\S}$ Mark E. Lowe, ${ }^{*}$ Satdarshan P. Monga,,\|\| Gustavo K. Rohde, ${ }^{\Uparrow}$ Farzad Esni, ${ }^{\dagger}$ and Sohail Z. Husain ${ }^{*}$

From the Department of Pediatric Gastroenterology, Hepatology, and Nutrition,* University of Pittsburgh School of Medicine, Pittsburgh; the Department of Surgery, ${ }^{\dagger}$ University of Pittsburgh, Children's Hospital of Pittsburgh of UPMC, Pittsburgh; the Department of Pathology, ${ }^{\ddagger}$ University of Pittsburgh, Children's Hospital of Pittsburgh of UPMC, Pittsburgh; the Department of Pathology, ${ }^{\S}$ University of Pittsburgh, Pittsburgh; the Biomedical and Electrical and Computer Engineering, " Center for Bioimage Informatics, Carnegie Mellon University, Pittsburgh; and the McGowan Institute for Regenerative Medicine," University of Pittsburgh, Pittsburgh, Pennsylvania

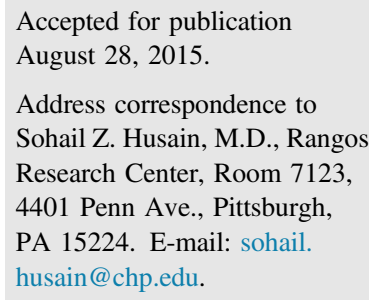

\begin{abstract}
The mechanisms by which drugs induce pancreatitis are unknown. A definite cause of pancreatitis is due to the antiepileptic drug valproic acid (VPA). On the basis of three crucial observations-that VPA inhibits histone deacetylases (HDACs), HDACs mediate pancreas development, and aspects of pancreas development are recapitulated during recovery of the pancreas after injury - we hypothesized that VPA does not cause injury on its own, but it predisposes patients to pancreatitis by inhibiting HDACs and provoking an imbalance in pancreatic recovery. In an experimental model of pancreatic injury, we found that VPA delayed recovery of the pancreas and reduced acinar cell proliferation. In addition, pancreatic expression of class I HDACs (which are the primary VPA targets) increased in the midphase of pancreatic recovery. VPA administration inhibited pancreatic HDAC activity and led to the persistence of acinar-to-ductal metaplastic complexes, with prolonged Sox9 expression and sustained $\beta$-catenin nuclear activation, findings that characterize a delay in regenerative reprogramming. These effects were not observed with valpromide, an analog of VPA that lacks HDAC inhibition. This is the first report, to our knowledge, that VPA shifts the balance toward pancreatic injury and pancreatitis through HDAC inhibition. The work also identifies a new paradigm for therapies that could exploit epigenetic reprogramming to enhance pancreatic recovery and disorders of pancreatic injury. (Am J Pathol 2015, 185: 3304-3315; http://dx.doi.org/10.1016/j.ajpath.2015.08.006)
\end{abstract}

We have recently gained a greater appreciation that the pancreas has a tremendous ability to recover and regenerate after injury. ${ }^{1,2}$ In experimental models of pancreatitis, such as by chemical induction with the cholecystokinin analog caerulein, there is dropout of more than half of the pancreatic parenchyma. ${ }^{3}$ A week after the insult, however, near-total pancreatic reconstitution is observed. ${ }^{3,4}$ Given these observations, understanding the regulatory signals that allow the pancreas to recover from injury could provide a clue to devising effective therapies for pancreatitis.
The clinical burden of pancreatitis is high. It ranks as the third most common inpatient gastrointestinal diagnosis in the United States and accounts for $>200,000$ hospitalizations annually. ${ }^{5,6}$ Approximately $10 \%$ to $15 \%$ of patients

Supported by NIH grants DK083327, DK093491, and DK03002 (S.Z.H.), the National Pancreas Foundation grant (J.F.E.), NIH mentored training grant K12 HD052892 08 (J.F.E.), and NIH training grant T32 DK071492 07 (M.E.L. and J.F.E.).

F.E. and S.Z.H. contributed equally as senior authors.

Disclosures: S.P.M. is a consultant for Abbvie but has no conflict relevant to the current study. 
develop severe pancreatitis, and one-quarter of severely affected patients will die. Causes of pancreatitis include a genetic predisposition, pancreaticobiliary anomalies, and environmental exposures. Little is known about the mechanisms by which medication exposure, a common environmental cause, induces pancreatitis.

The antiepileptic medication valproic acid (VPA) is a definite cause of pancreatitis that is associated with a clinically severe disease course. ${ }^{7,8}$ However, long-term administration of VPA (for 1 year) to mice and dogs had no effect on the pancreas, and in rats, there were only subtle microscopic findings of pancreatic lobular atrophy and vacuolization. ${ }^{9}$ Short-term exposure to VPA by itself failed to induce any pancreatic changes in these animals. It is unclear whether VPA in combination with other inducers would predispose to pancreatitis.

VPA is a carboxylic acid analog of valeric acid, which is derived from the plant Valeriana officinalis. ${ }^{10,11}$ It is used as an antiseizure medication and a medication for pain modulation. The mechanism of VPA's antiseizure properties are thought to be due to its ability to increase $\gamma$-amino butyric acid levels by augmenting the activity of glutamate decarboxylase. However, VPA has also long been known to inhibit a group of epigenetic proteins - the histone deacetylases (HDACs). ${ }^{10,11}$ HDACs were named based on their ability to remove acetyl groups from lysine residues on histone tails. ${ }^{11}$ Deacetylation of histones compacts DNA in the nucleosome and thus represses gene expression. Indeed, the ability of HDAC inhibitors (HDACis), including VPA, to modulate gene expression has recently been exploited in cancer, neurodegenerative diseases, and stem cell biology. ${ }^{12-15}$ HDACs were recently found to be upregulated in the embryonic pancreas, ${ }^{16}$ and HDACi administration during development shifted the lineage of pancreatic precursors from an acinar to islet cell phenotype. ${ }^{17}$ This finding suggested that HDACs might be important for orchestrating the signals that dictate pancreatic cell differentiation. Furthermore, aspects of embryonic development of the pancreas are recapitulated during recovery and regeneration after pancreatic injury. ${ }^{4,18-20}$ On the basis of these three crucial observations-that VPA inhibits a class of epigenetic factors (the HDACs), HDACs modulate pancreas development, and expression patterns of pancreas development are reactivated during recovery of the pancreas after injury-we hypothesized that VPA predisposes patients to pancreatitis by inhibiting HDACs and thereby provoking an imbalance in pancreatic recovery.

We found that VPA impedes pancreatic recovery by reducing acinar cell proliferation and causing the persistence of acinar-to-ductal metaplasia (ADM) complexes and early embryonic programming as well as delaying acinar redifferentiation. Using a non-HDACi analog as a negative control, we deduced that the effect of VPA on recovery appears to be mediated through its inhibition of HDACs.

\section{Materials and Methods}

\section{Reagents and Animals}

Caerulein was purchased from Sigma-Aldrich (St. Louis, MO). Six-week-old male Swiss Webster mice (Charles River, Wilmington, MA) weighing 20 to $25 \mathrm{~g}$ were fed standard laboratory chow with free access to water. All animal experiments were performed using a protocol approved by the University of Pittsburgh Institutional Animal Care and Use Committee.

Pancreatic injury and acinar dropout were reproducibly modeled in mice by a pancreatitis induction method using the cholecystokinin analog caerulein at a hyperstimulatory dose of $50 \mu \mathrm{g} / \mathrm{kg}$ (Figure 1A). ${ }^{4}$ Mice received 8 hourly i.p. caerulein injections on 2 consecutive days (days -2 and -1 ). VPA and valpromide (VPD) (Sigma-Aldrich) were prepared at a concentration of $125 \mathrm{mg} / \mathrm{kg}$ and injected i.p. every 12 hours after caerulein injury, starting on day 0 . Dosing and frequency of VPA were based on published reports in mice to provide VPA serum levels in the approximate range of 15 to $40 \mu \mathrm{g} / \mathrm{mL} .^{21,22}$ Two hours before euthanization, mice were injected i.p. with a single dose of $0.9 \mathrm{mg}$ of BrdU to track cell proliferation. Animals were euthanized by $\mathrm{CO}_{2}$ asphyxiation and cervical dislocation on days 1,3 , and 7 after the last day of caerulein injections. Fragments of pancreas tissue were placed in $4 \%$ paraformaldehyde for hematoxylin and eosin (H\&E) staining, immunofluorescence (IF), and immunohistochemistry (IHC), flash frozen for Western immunoblotting (WB) or acetylase activity assays, or processed for RNA extraction.

Antibodies used included the following: goat antiE-cadherin (1:200 IF; R\&D Systems, Minneapolis, MN; catalog no. AF748), rat anti-E-cadherin (1:200 IF; Invitrogen, Carlsbad, CA; catalog no. 13-1900), rabbit anti-pan-CK (1:100 IF; Dako, Carpinteria, CA; catalog no. z0622), rabbit anti-Sox9 (1:1000 IF; Millipore, Billerica, MA; catalog no. AB5535), rabbit antiamylase (1:300 IF; Sigma-Aldrich; catalog no. A8273), goat antiamylsae (1:250 IF; Santa Cruz Biotech, Santa Cruz, CA; catalog no. Sc-12821), rabbit anti-histone 3 (H3) (1:1000 WB; Cell Signaling, Danvers, MA; catalog no. 9715), rabbit anti-acetyl-H3 (1:1000 WB; Abcam, Cambridge, MA; catalog no. ab47915), rat anti-BrdU (1:100 IF; Abcam; catalog no. ab6326), mouse anti-HDAC1 (1:500 WB; Cell Signaling; catalog no. 5356), rabbit anti- $\beta$-catenin (1:150 IHC; Santa Cruz Biotech; catalog no. sc-7199), mouse anti- $\beta$-catenin (1:150 WB; BD Biosciences, San Jose, CA; catalog no. BD610154), and rabbit anti-TCF4 (1:500 WB; Cell Signaling; catalog no. 2569).

\section{Histologic Preparation and Generation of Digital Images for Analysis}

Pancreatic sections were processed by a single facility (the Histology Core at the Children's Hospital of Pittsburgh) to standardize the $\mathrm{H} \& \mathrm{E}$ staining process for each of the 
A

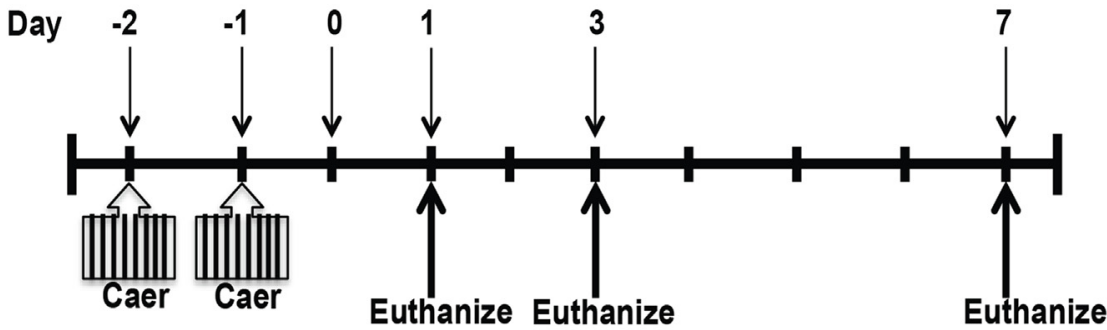

Figure 1 Valproic acid (VPA) limits pancreatic recovery after injury. A: Scheme for injury induction and for tracking recovery. Eight hourly injections of caerulein (Caer) were given on 2 consecutive days (days -2 and -1 ), and recovery

B

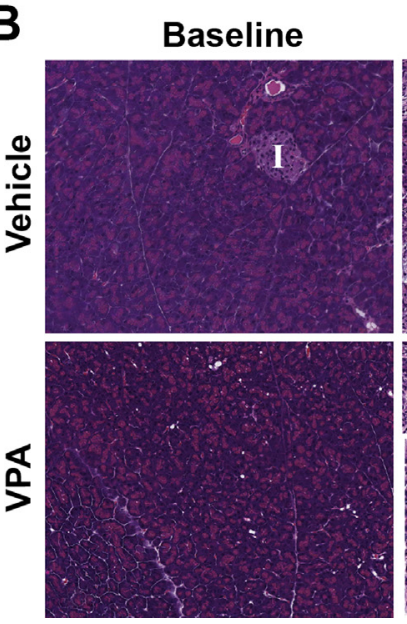

Day 3

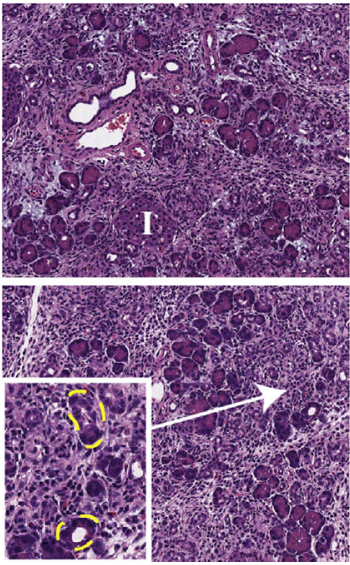

Day 7

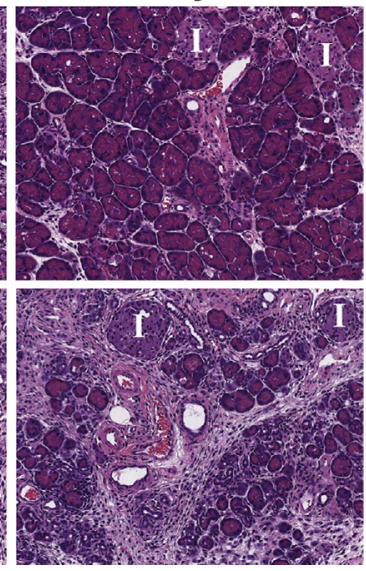
was subsequently monitored for 7 days. VPA was administered throughout the recovery period, as described in the Materials and Methods. B: Hematoxylin and eosin-stained sections of pancreas reveal recovery of acinar content in vehicle-treated control mice by day 7 . Inset shows ADMS (outlined in yellow) at high power. The VPA-treated mice have reduced recovery. Original magnification: $\times 20$ (B); $\times 40$ (inset). I, islet.

pancreatic samples. High-resolution digital images were acquired using an Aperio whole slide scanner (Aperio ePathology Solutions, Leica Microsystems, Inc., Buffalo Grove, IL) at a magnification of $\times 400$, which yielded a file size of 750 to 900 megabytes. The spatial resolution of images from the scanner at this magnification was $0.14 \mu \mathrm{m} /$ pixel. To accurately quantify acinar cell content in $\mathrm{H} \& \mathrm{E}$ pancreas sections, we applied a previously published computer-learning algorithm. ${ }^{3}$ Briefly, this algorithm consists of two main stages: training and segmentation. In the training stage, expert-labeled images are given to the algorithm for distinguishing acinar cells from other cell types in the tissue. After normalization of these images, the algorithm extracts $3 \times 3$-pixel neighborhood intensities for each pixel in color for three different resolutions of the original image. This multiple resolution information allows the algorithm to evaluate spatial information about each pixel's neighborhood. Using this information, the algorithm trains multiple classifiers for each resolution. Once the classifiers are trained, second stage segmentation can be applied to test images of pancreas tissue where segmentations are obtained by classifying every single pixel using each of the classifiers. The algorithm then takes a majority vote (over different image resolutions) within spatially predefined local windows. Segmentation results are then analyzed to quantify the acinar content, which is the percentage of segmented acinar regions over the entire pancreas tissue for each image. In addition, images from day 1 were also scored for acinar injury based on a previously published scoring system. ${ }^{23}$

\section{Quantitative Analysis of ADM from H\&E Sections}

$\mathrm{H} \& \mathrm{E}$-stained slides were analyzed for ADM complexes in a blinded fashion (J.F.E., A.W.D) by manual counting of 10 random high-powered fields (HPFs) from pancreata of at least three mice from each time point and condition. ADMs were defined based on a compilation of criteria, ${ }^{24-26}$ including i) luminal dilatation (with or without intraluminal cell debris), ii) cuboidal acinar cells (exhibiting higher nuclear to cytoplasmic ratios than normal acinar

Table 1 List of Histone Deacetylase Primers Used

\begin{tabular}{lll}
\hline Gene & Qiagen QuantiTect Primer Assay & Catalog no. \\
\hline GAPDH & Mm_Gapdh_3_SG & QT01658692 \\
Hdac1 & Mm_Hdac1_1_SG & QT00282268 \\
Hdac2 & Mm_Hdac2_1_SG & QT01161657 \\
Hdac3 & Mm_Hdac3_1_SG & QT00103593 \\
Hdac4 & Mm_Hdac4_1_SG & QT00170436 \\
Hdac5 & Mm_Hdac5_1_SG & QT01040907 \\
Hdac6 & Mm_Hdac6_1_SG & QT00118349 \\
Hdac7 & Mm_Hdac7_1_SG & QT00147658 \\
Hdac8 & Mm_Hdac8_1_SG & QT00302428 \\
Hdac9 & Mm_Hdac9_1_SG & QT01054368 \\
Hdac10 & Mm_Hdac10_1_SG & QT00143395 \\
Hdac11 & Mm_Hdac11_1_SG & QT00142058 \\
\hline
\end{tabular}


A

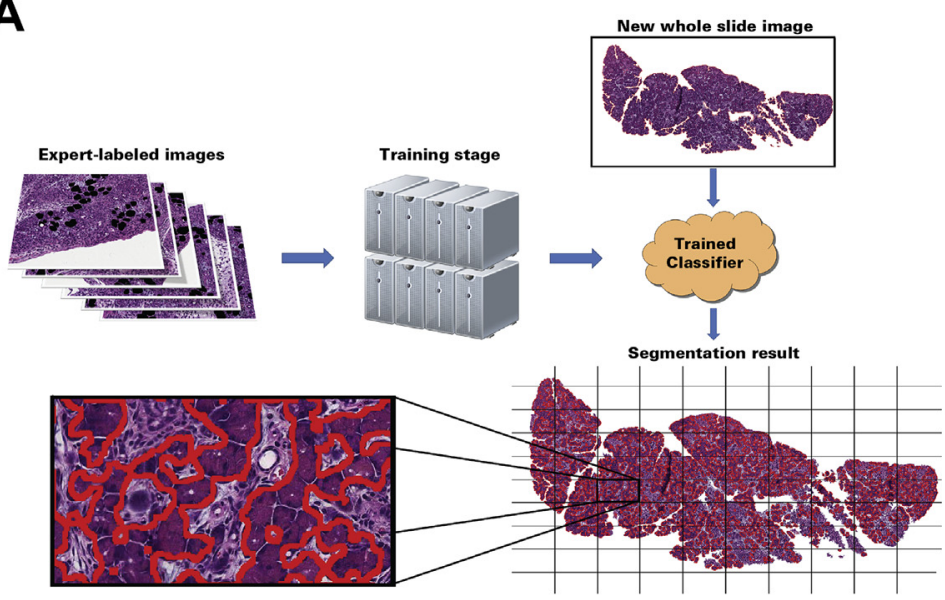

B

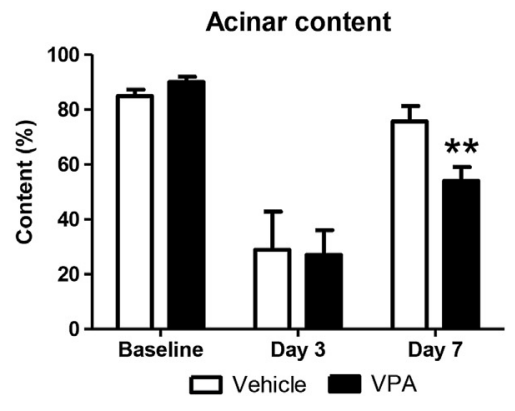

Figure 2 Using a machine-learning algorithm to objectively quantify acinar content, valproic acid (VPA) prevents acinar cell reconstitution in the recovering pancreas. A: Scheme for computer learning. A trained classifier is first generated and then validated. The validated classifier can be used to quantify acinar cell content in hematoxylin and eosin-stained pancreas sections. B: Quantification of acinar cell content from whole slides scanned at $60 \times$ magnification. $n=3$ to 5 mice. ${ }^{* *} P<0.01$, relative to the vehicle-treated group.

cells) within the acinar structures, iii) loss of zymogen granules, iv) cytoplasmic vacuolization, and v) interlobular edema. The mean number of ADMs was calculated and represented as the means \pm SD for each time point and condition.

\section{Real-Time Quantitative PCR}

Real-time quantitative PCR and WB were performed from baseline (noninjured) or injured pancreata from the different experimental conditions as outlined above. For quantitative RT-PCR, mRNA isolation and subsequent cDNA synthesis were performed using the mMACS One-Step cDNA kit (Miltenyi Biotec, Cambridge, MA; catalog no. 130-091902) according to the manufacturer's instructions. The PCR primers (QuantiTect Primer Assays) were purchased from Qiagen (Valencia, CA). Reactions were performed with PerfeCTa SYBR Green SuperMix for IQ (Quanta Biosciences, Gaithersburg, MD; catalog no. 95053) on a Bio-Rad IQ5 Instrument (Bio-Rad, Hercules, CA). Reactions were performed at least in triplicate, and specificity of the amplified products was determined by melting peak analysis. Quantification for each gene of interest was performed with the 2- $\Delta \Delta \mathrm{Ct}$ method. Quantified values were normalized against the housekeeping gene $G A D P H$, which proved to be equally expressed across the samples. Primers are listed in Table 1.

\section{Western Blotting}

For WB, pancreas tissues were lyzed in NE-PER Nuclear and Cytoplasmic extraction reagent supplemented with protease inhibitors (Thermo Scientific, Waltham, MA; catalog no. 78835). Protein quantification was performed with a Pierce BCA Protein Assay Kit (Thermo Scientific; catalog no. 23227). Protein lysates were separated on $4 \%$ to
$20 \%$ gradient SDS-polyacrylamide gel electrophoresis gels (Mini-PROTEAN TGX Gels; BioRad; catalog no. 456-1094) and transferred onto $0.2-\mathrm{mm}$ nitrocellulose membranes using a Mini Trans-Blot electrophoretic apparatus (BioRad). All buffers were purchased from BioRad. Staining for HDAC1 was performed using staining of H3 with an anti-H3 antibody as a loading control.

\section{Measurement of Deacetylase Activity}

Nuclear extracts were processed to measure deacetylase activity using the EpiQuik HDAC Activity/Inhibition Assay Kit (Epigentek Group Inc., Farmingdale, NY; catalog no. P-4002) from flash frozen pancreas tissue, according to the manufacturer's instructions.

\section{Immunoprecipitation}

Co-precipitation studies were performed with $20 \mu \mathrm{g}$ of isolated nuclear protein from each animal group at specified time points. Co-precipitation experiments were performed at least two times on two to three animals. The antibody used for immunoprecipitation was rabbit TCF4 (Cell Signaling Technology, Danvers, MA; catalog no. 2569). Western analysis was performed with mouse anti- $\beta$-catenin (BD Biosciences; catalog no. BD610154) and rabbit TCF4.

\section{IF and Quantification Analysis}

Tissue processing, immunostaining, and quantitative analyses were performed as described previously. ${ }^{27}$

\section{Statistical Analysis}

Results are expressed as means $\pm \mathrm{SD}$, unless otherwise stated. Statistical significance between two groups was 


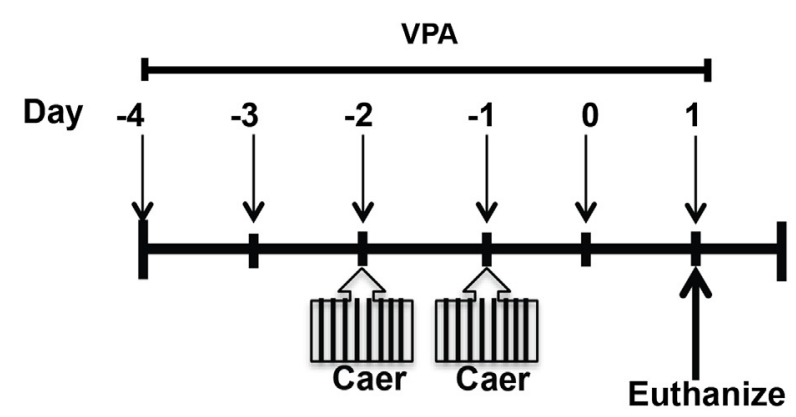

B
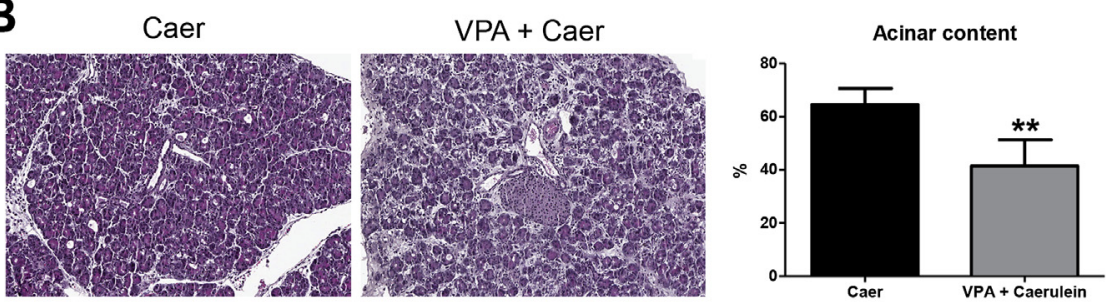

C
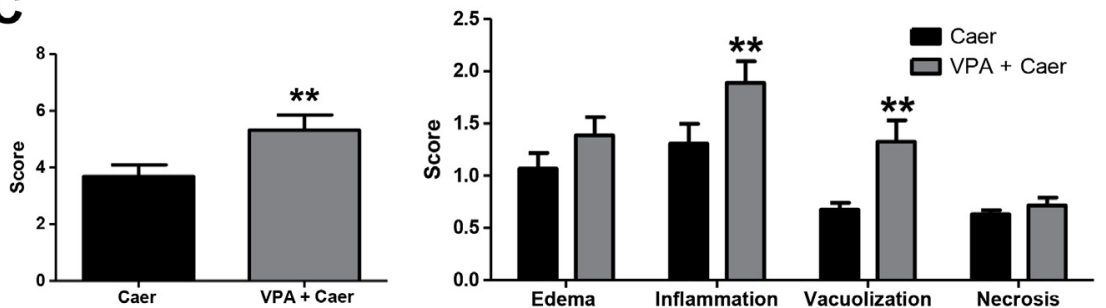

Figure 3 Valproic acid (VPA) pretreatment induces a net increase in pancreatic injury after caerulein (Caer) hyperstimulation. A: Scheme for injury induction. VPA was administered starting 2 days (days -4 and -3 ) before induction of injury with Caer, as described in the Materials and Methods. Eight hourly injections of Caer were given on 2 consecutive days (days -2 and -1 ), and injury assessed at day 1. B: Representative image of pancreas tissue at day 1 after injury. Acinar content measured by machine learning algorithm. C: Histologic scoring of pancreatic sections stained with hematoxylin and eosin. Severity score is combination of edema, inflammation, necrosis, and vacuolization. $n=3$ animals per group. ${ }^{* *} P<0.01$ relative to the control group. Original magnification: $\times 20(B)$. determined using a $t$-test, and a one-way analysis of variance was used for comparisons among three or more groups. Significance was defined as a $P<0.05$.

\section{Results}

\section{VPA Limits Pancreatic Recovery after Injury}

To examine pancreatic recovery, we first induced injury using a caerulein hyperstimulation model of pancreatitis in mice. ${ }^{28-31}$ Caerulein is an oligo-peptide analog of cholecystokinin, a hormone that stimulates the secretion of pancreatic enzymes from the acinar cell. ${ }^{32,33}$ Hourly i.p. injections of supraphysiologic concentrations of caerulein (50 $\mathrm{mg} / \mathrm{kg}$ ) caused a consistent and relatively uniform interstitial pancreatitis in mice (Figure 1). Pancreas tissue harvested from caerulein-treated mice revealed homogeneous acinar cell injury that was marked by vacuolization of acinar cells and interstitial edema at day 1 (data not shown). ${ }^{3}$ Injury peaked by day 3 (Figure 1B) with histologic evidence of severe acinar cell loss and replacement of many acinar cells by transient duct-like tubular complexes called ADM (Figure 1B). One week after injury, the pancreatic exocrine compartment markedly recovered, closely resembling a normal pancreatic histologic appearance (Figure 1B).

To study the effects of VPA on pancreatic recovery, we administered VPA every 12 hours after the induction of pancreatic injury. Day 0 referred to the first day after the last caerulein injection and also the first day of VPA administration. In the first experimental condition, we found that mice given VPA alone for 7 days (ie, without caerulein) had no appreciable changes in acinar content and no detectable injury compared with baseline negative control mice (ie, given phosphate-buffered saline, the VPA vehicle) (Figure 1B). Mice treated with VPA after caerulein-induced injury had similar injury at day 3 compared with vehicle-treated mice that received caerulein. However, at day 7 after caerulein administration, VPA-treated mice had a delay in pancreatic recovery, marked by a reduced number of acinar cells and a sustained presence of ADMs that were accompanied by stromal edema and inflammation (Figure 1B). By contrast, the vehicle-treated mice had near-complete recovery by day 7 .

To more accurately quantify acinar cell content in $\mathrm{H} \& \mathrm{E}$ pancreas sections, we applied our previously published methods for quantifying acinar content from whole slide digital images. ${ }^{3}$ Briefly, a computer identified acinar cells by analyzing labeled images of acinar cells provided by experts and then generated an algorithm that could be applied to new images of $\mathrm{H} \& \mathrm{E}$-stained pancreatic sections (Figure 2A). We first observed that VPA administration by itself to healthy noninduced mice had no visible effect on pancreatic histologic appearance. In particular, with use of the machine learning segmentation tool, there was no objective difference in acinar content compared with vehicle-treated mice. Both had similar 
A<smiles>CCCC(CCC)C(=O)O</smiles>

B

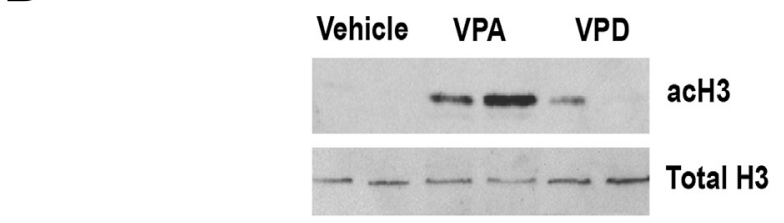

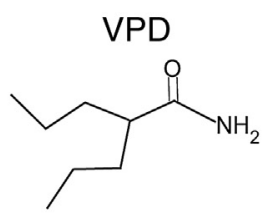

C

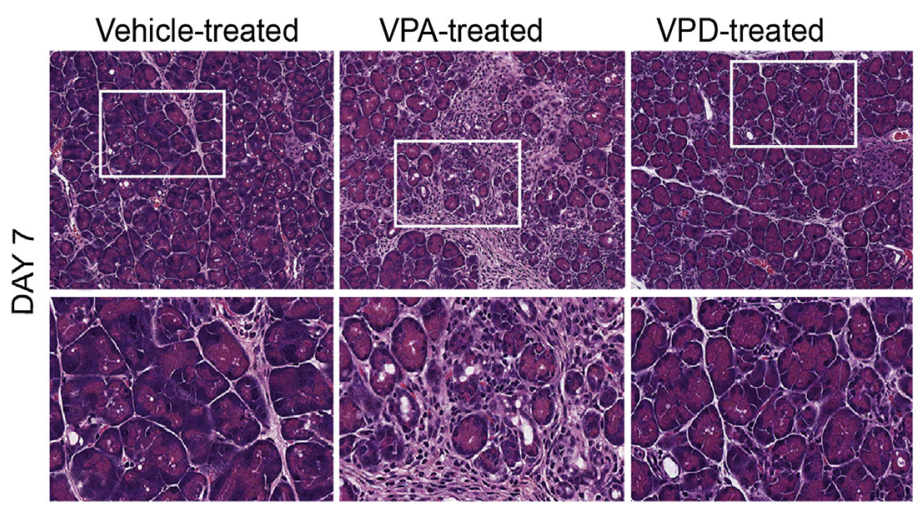

D
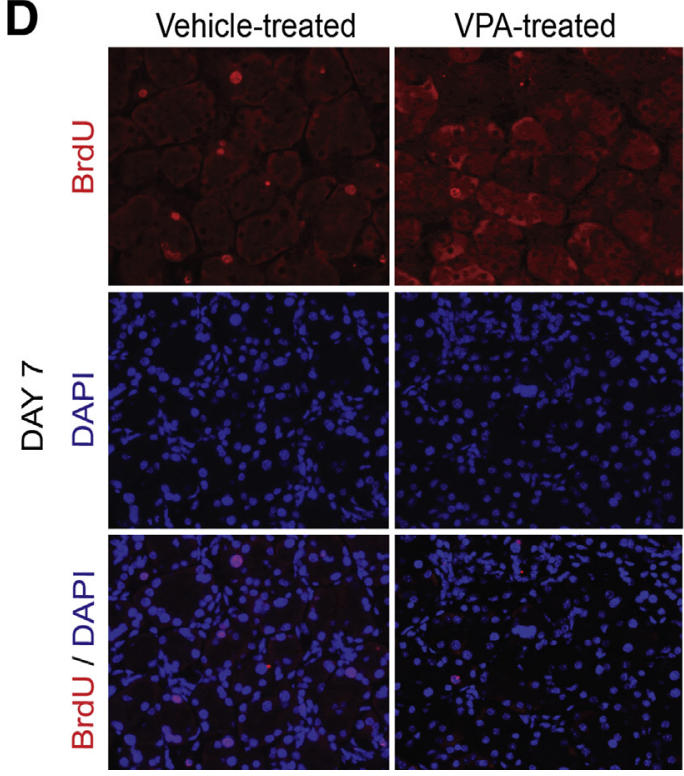

VPD-treated

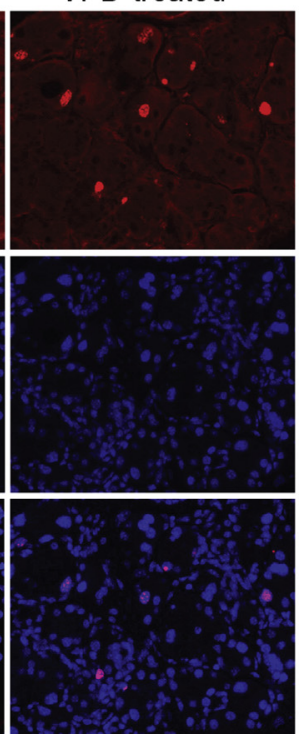

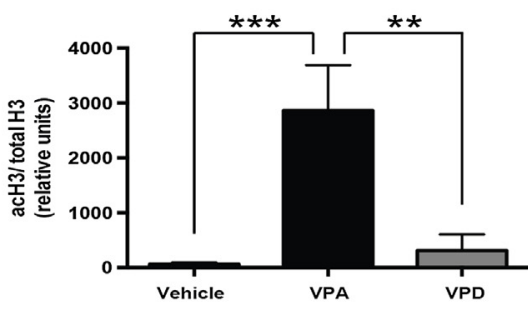
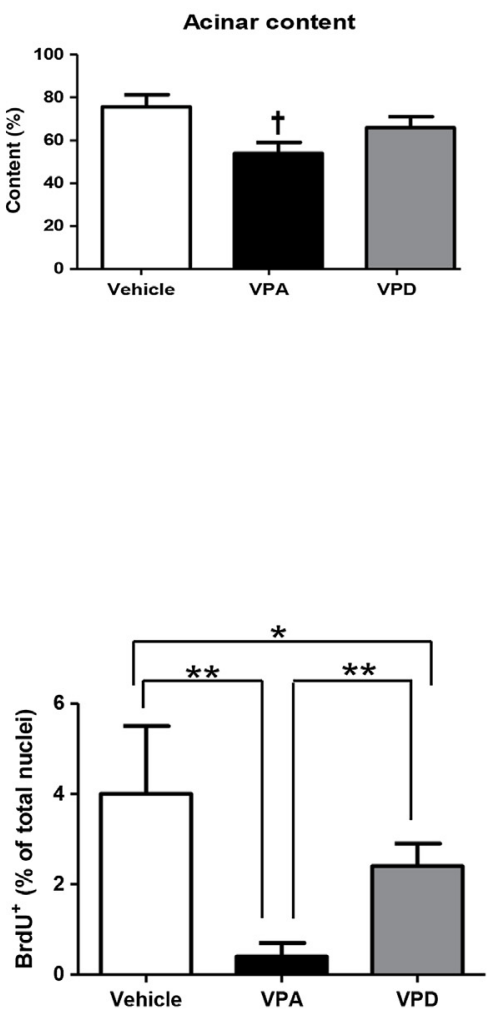

Figure 4 A carboxamide derivative of valproic acid (VPA) that has markedly reduced histone deacetylase inhibition (HDACi) does not affect pancreatic recovery, and HDACi with VPA reduces the acinar cell proliferation that is induced during recovery. A: Chemical structures for VPA and valpromide (VPD). B: Western blotting and densitometry from acetylation of histone $3(\mathrm{acH} 3)$ compared with total $\mathrm{H} 3$ from pancreatic nuclear extracts of mice receiving vehicle, VPA, or VPD. C: Pancreatic hematoxylin and eosin of the three groups along with quantification by machine learning. Boxed areas are shown below at higher magnification. D: Immunofluorescence labeling of $\mathrm{BrdU}$ in representative pancreas sections from the three groups at day 7 . Quantification of BrdU ${ }^{+}$nuclei versus acinar nuclei stained by DAPI. $n=4$ animals from each group (B); $n=3$ to 5 animals per group (C); $n=3$ animals per group (D). ${ }^{*} P<0.05, * * P<0.01$, and ${ }^{*} * * P<0.001$ (B and $\left.\mathbf{D}\right) .{ }^{\dagger} P<0.05$ relative to the vehicle- and VPD-treated groups. Original magnification: $\times 20(\mathbf{C}$, top row); $\times 40(\mathbf{C}$, bottom row, and $\mathbf{D})$.

acinar content values at $83 \% \pm 9 \%$ and $85 \% \pm 10 \%$ (Figure 2B).

VPA, however, retarded pancreatic recovery when it was given after pancreatitis. At day 3 after caerulein administration, the acinar content of both groups of mice (vehicle and VPA treated) was similarly reduced $(29 \% \pm 10 \%$ and $26 \% \pm 8 \%$, respectively). By day 7 , on the other hand, acinar cell content in vehicle-treated mice given caerulein returned to $74 \% \pm 9 \%$ 


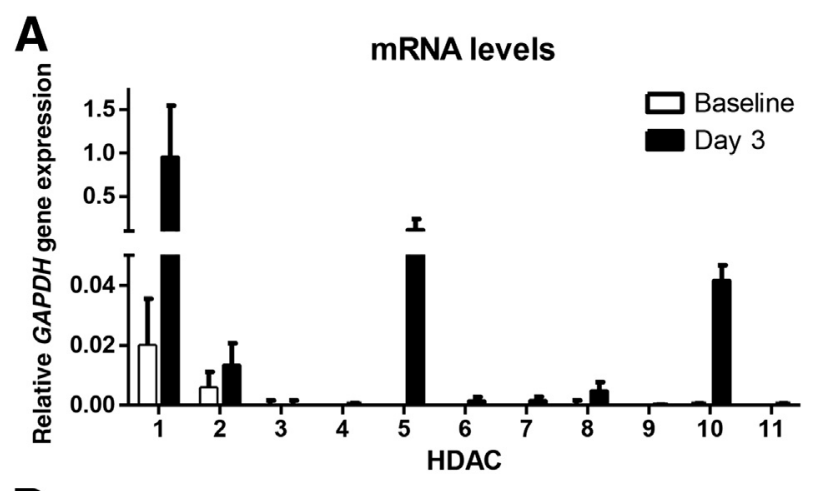

B
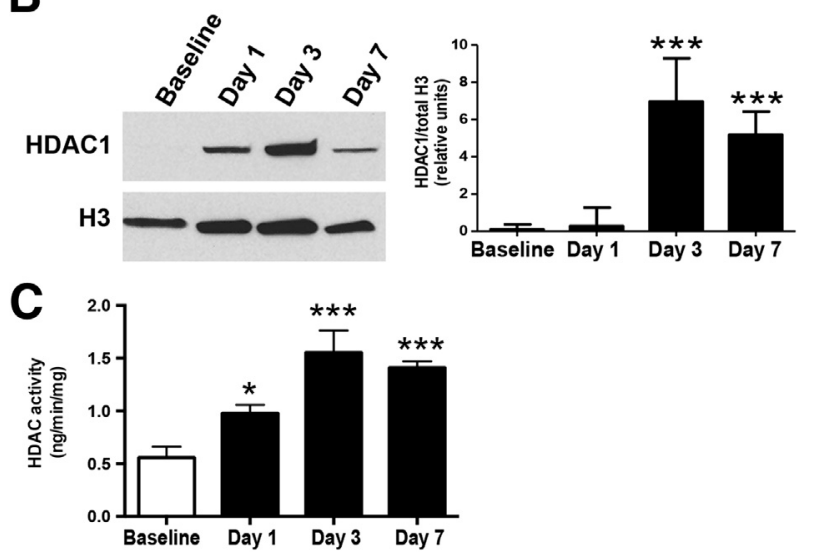

Figure 5 Histone deacetylases (HDACs) are up-regulated within the pancreas during recovery after injury. A: Expression of the class 1 HDACs at baseline and day 3. B: Western blotting for HDAC1 from pancreatic nuclear extracts, normalized to histone 3. C: Nuclear HDAC activity. $n=5$ animals per group $(\mathbf{A}-\mathbf{C}) .{ }^{*} P<0.05,{ }^{* *} P<0.001$ relative to baseline (B and $\left.\mathbf{C}\right)$.

whereas the VPA-administered caerulein-treated mice recovered only half of the pancreatic acinar content $(54 \% \pm 10 \%$, $P<0.05$ ) (Figure 2B). These data suggest that VPA administration during pancreatic recovery reduces the normal regenerative capacity of pancreatic acinar cells.

In addition, if VPA was administered before the initiation of injury (ie, 2 days before caerulein) (Figure $3 \mathrm{~A}$ ), there was an increase in the amount of injury seen at day 1 (Figure 3B). The higher level of injury was manifested by greater acinar cell loss (Figure 3B), a heightened inflammatory response, and more vacuolization (Figure 3C). Taken together, these data suggest that VPA does not cause pancreatitis but instead alters the progression of pancreatitis.

To deduce whether the HDACi action of VPA contributes to the delay in acinar recovery after caerulein administration, we gave a parallel group of mice a VPA analog, VPD, which acts similar to VPA ${ }^{21,34,35}$ except that it has only $10 \%$ to $19 \%$ of the HDAC inhibitor activity of VPA (Figure 4A). To confirm the relative HDACi activity of VPA and VPD in the pancreas, we evaluated the acetylation status of $\mathrm{H} 3$ from pancreatic nuclear fractions of mice treated with vehicle, VPA, or VPD at day 7 after caerulein administration. VPA and VPD treatment caused a 45-fold and fivefold increase, respectively, in $\mathrm{H} 3$ acetylation in mice above baseline vehicle treatment; thus, the relative increase in $\mathrm{H} 3$ acetylation with VPD was only $11 \%$ of the increase with VPA (Figure $4 B$ ). The higher amount of $\mathrm{H} 3$ acetylation at day 7 in VPA-treated mice, indicating $\mathrm{HDACi}$, correlated with the greater delay in acinar cell regeneration (Figure 4C). Taken together, these data suggest that VPA reduces pancreatic regeneration through inhibition of HDAC activity.

To examine whether VPA affects acinar cell proliferation during pancreatic recovery, BrdU was injected 2 hours before euthanizing the animals. BrdU incorporation with this protocol would identify cells that are proliferating at the time of euthanization. At day 7, in all treatment animals, the predominant cell type containing BrdU incorporation is the acinar cell. VPAtreated animals had a 10-fold reduced level of acinar cell proliferation compared with vehicle-treated animals at day 7 (Figure 4D). VPD-treated animals had a modest 1.6-fold reduced level of acinar cell proliferation. These data suggest that VPA limits acinar cell proliferation through an HDACmediated pathway.

\section{HDACs Are Up-Regulated during Pancreatic Recovery after Injury}

Having implicated a role for HDACs (using VPA compared with VPD) in pancreatic recovery, we next examined whether pancreatic HDAC expression is increased during recovery. There are 18 different HDACs, divided into four classes. ${ }^{12}$ Class I (HDAC1, 2, 3, and 8), class II (HDAC4, 5, 6, 7, 9, and 10), and class IV (HDAC11) HDACs share homology, require $\mathrm{Zn}^{2+}$ as a cofactor, and are termed the classic HDACs. The atypical class III HDACs are structurally different and use nicotinamide adenine dinucleotide as a cofactor. By real-time quantitative PCR from pancreas tissue in caerulein-treated mice, we found differential expression patterns of the classic HDACs between baseline and day 3 (Figure 5A). HDAC3, 4, 6, 7, 9, and 11 expression levels were unchanged, whereas levels of HDAC1, 2, 5,8 , and 10 were increased. The most highly expressed HDAC at day 3 was the class I HDAC1 (50-fold increase above baseline).

We next examined expression of HDAC1 protein levels by WB of nuclear extracts from pancreas tissue (Figure 5B). Protein isolation was performed at day 1 , day 3 , and day 7 after injury and from baseline control mice and compared to total $\mathrm{H} 3$ expression. HDAC 1 expression was low at baseline and appeared to increase with injury. It peaked at day 3 but remained somewhat elevated at day 7 (Figure 5B).

We next assayed global pancreatic nuclear HDAC activity after caerulein hyperstimulation (Figure 5C). HDAC activity increased after pancreatic injury, also peaking at day 3 , and remained elevated at day 7 . These data, taken together with the H3 acetylation at day 7 (Figure 4B), suggest that HDAC activity is increased after injury.

\section{HDACs Are Crucial for Proper Recovery and Redifferentiation of Mature Acinar Cells from ADMs}

After acute injury and inflammation during pancreatitis, many of the surviving acinar cells dedifferentiate into 
A
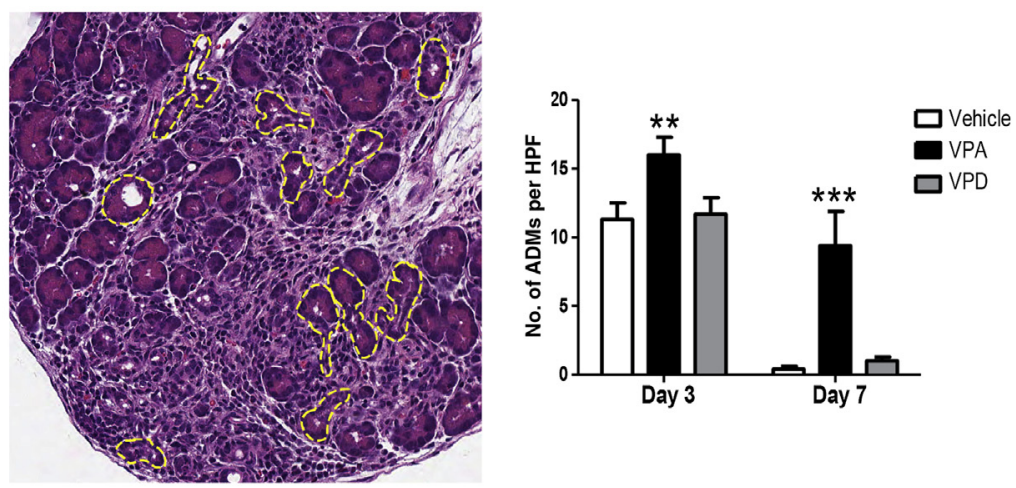

B
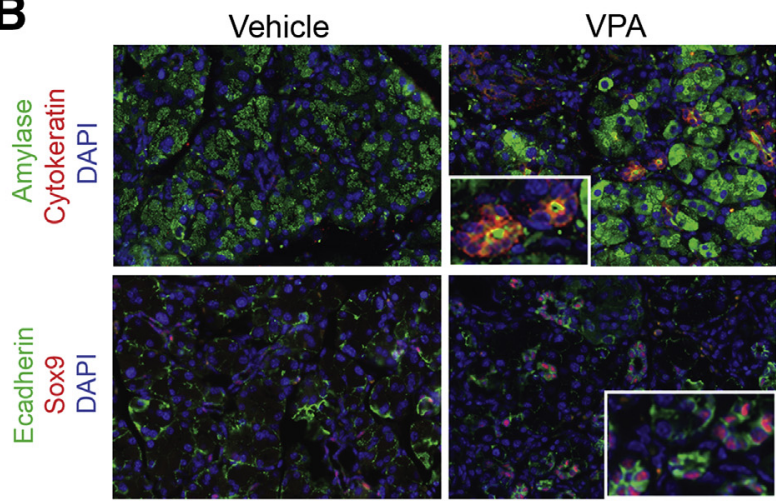

C
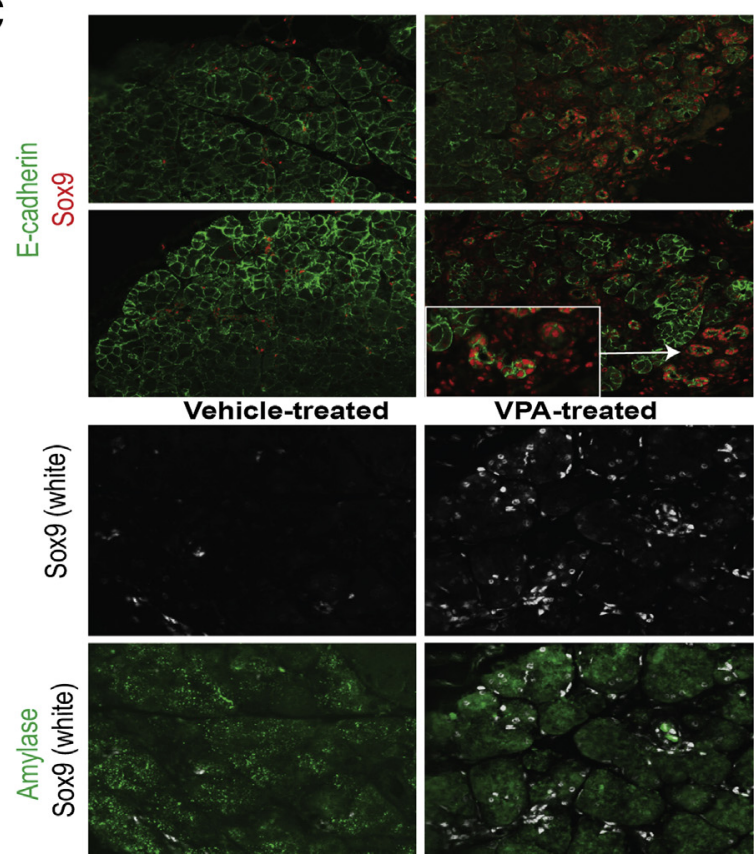

VPA-treated

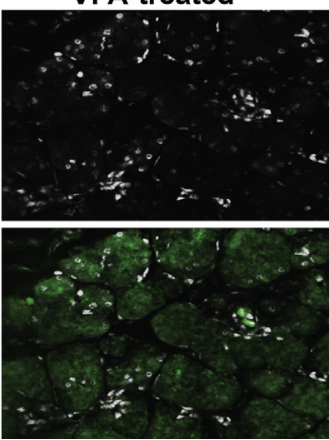

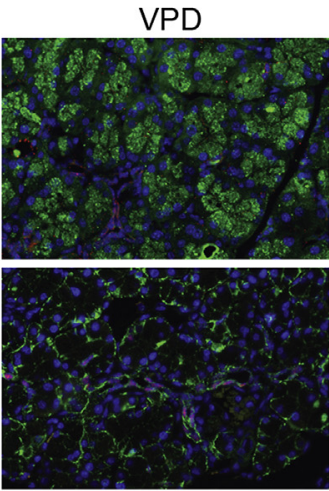

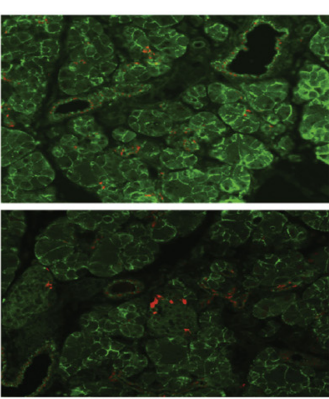

VPD-treated

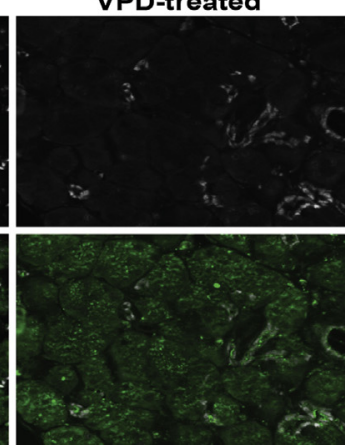

Figure 6 Histone deacetylase inhibition (HDACi) with valproic acid (VPA) causes persistence of acinar-to-ductal metaplasia (ADM) complexes during pancreatic recovery. A: Representative hematoxylin and eosin-stained section from a vehicle-treated mouse on day 3 with ADMs outlined by a dashed yellow line as discrete structures and the quantification of the number of ADMs. B: The ADMs shown at day 7 among the three groups are tubular structures that express acinar (amylase) and ductal (cytokeratin, Sox9) markers. Insets are a magnified image of ADMs. C: Sox9 staining of vehicle-, VPA-, and valpromide (VPD) - treated animals. Representative images are shown. In VPAtreated animals, Sox9 expression persists in ADMs and some acinar cells. $n=3$ animals per group (A). $* * P<0.01, * * * P<0.001$ relative to the vehicletreated group (A). Original magnification: $\times 40$ (A, and insets in B and C); $\times 20$ (B and C). HPFs, high-powered fields.
ADMs, which are metaplastic structures that are thought to serve as facultative progenitors during pancreatic regeneration. The dedifferentiated acinar cells (or ADMs) assume an altered gene expression that is reminiscent of pancreatic embryonic precursors. ${ }^{4,18,19}$ During this transient state, acinar-specific expression of digestive enzymes decreases, and there is a concomitant increase in duct-specific markers, including cytokeratin and the transcription factor Sox 9. ${ }^{36-39}$
The epithelial cell membrane marker E-cadherin is also transiently diminished in these ADMs. ${ }^{40}$ During recovery from caerulein pancreatitis, ADMs are prominent at day 3 but diminish by day 7 , coincident with reconstitution of the pancreatic parenchyma to a near-normal histologic appearance (Figure 1B). At day 3 in H\&E-stained sections from vehicle- and VPD- treated animals, there were similar numbers of ADMs in both groups (12 \pm 1 ADMs per HPF; 


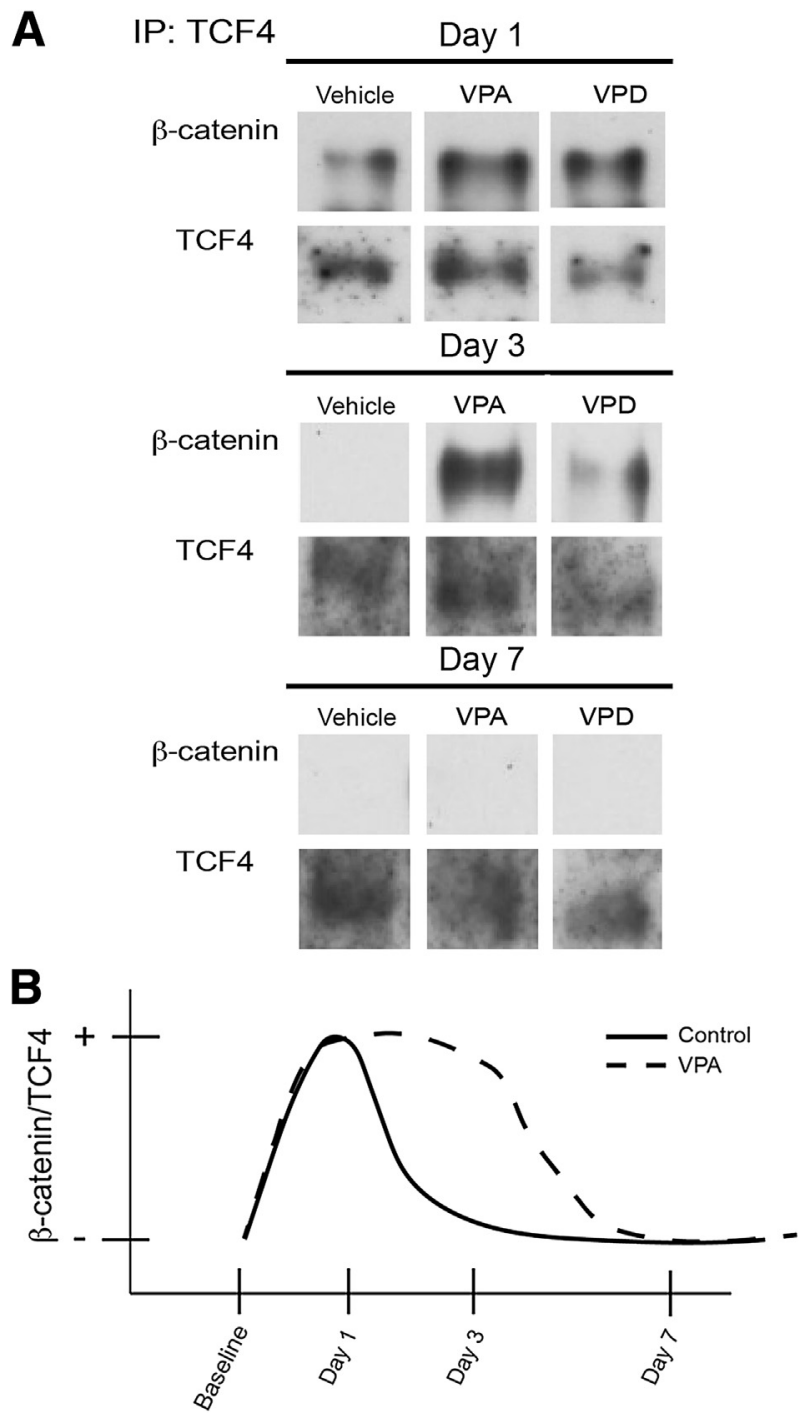

Figure 7 Valproic acid (VPA) modulates $\beta$-catenin nuclear translocation during pancreatic recovery. A: Co-immunoprecipitation (IP) with TCF antibody reveals that VPA enhances $\beta$-catenin/TCF4 interaction at day 3 after injury. B: Summary scheme depicting $\beta$-catenin-TCF4 interactions for animals treated with vehicle, VPA, and valpromide (VPD) after caerulein hyperstimulation. VPA prolongs the nuclear $\beta$-catenin/TCF4 interaction at day 3 .

Figure 6A). At day 3 the pancreas from VPA-treated animals, however, had a higher number of ADMs $(16 \pm 2$ ADMs per HPF; $P<0.01$ ). At day 7 , in both vehicle- and VPD-treated animals, there were few ADMs per HPF remaining $(1 \pm 1 \mathrm{ADM}$ per $\mathrm{HPF})$. By contrast, at day 7 , ADMs continued to be elevated to a large extent in VPAadministered caerulein-treated mice $(9 \pm 3$ ADMs per HPF; $P<0.001)$. These structures at day 7 in the VPA-treated group co-expressed amylase and cytokeratin and had Sox9 expression (Figure 6B), thus confirming their identity as ADMs.

Sox9 has been implicated in the formation of ADMs and the maintenance of an undifferentiated cell phenotype. ${ }^{38,41}$ At day 7 in vehicle- and VPD- treated animals, Sox9 expression was limited to ductal cells (Figure 6C), whereas E-cadherin staining predominated in acinar cells. In contrast, animals administered VPA continued to have increased Sox 9 expression in ADMs and immature acinar cells. These data demonstrate that VPA, but not its non-HDACi analog VPD, likely causes the persistence of ADMs during pancreatic recovery and suggest that the inhibition of HDACs prevent the metaplastic structures from redifferentiating into mature acinar cells.

\section{HDACs Control the Redifferentiation of ADMs to Acinar Cells through Wnt/ $\beta$-catenin}

$\beta$-catenin is a protein that in the basal state localizes to the acinar cell membrane and functions in cell-cell adhesions ${ }^{42}$ (Supplemental Figure S1). In baseline animals, $\beta$-catenin localized to the membrane in all treatment groups with undetectable nuclear localization by IHC (Supplemental Figure S1). However, there was a shift in $\beta$-catenin localization to the cytoplasm and nucleus on activation of embryonic wnt signaling, primarily within $\mathrm{ADMs}^{2,43,44}$ (Supplemental Figure S1). In fact, the Wnt/ $\beta$-catenin pathway's activation is required for $\mathrm{ADM}$ formation. ${ }^{20} \mathrm{We}$ found that VPA did not significantly affect the shift in $\beta$-catenin localization patterns seen during recovery (Supplemental Figure S1). Once in the nucleus, $\beta$-catenin binds the TCF/LEF DNA-binding proteins to induce gene expression. ${ }^{45,46}$ By immunoprecipitation of TCF/LEF member
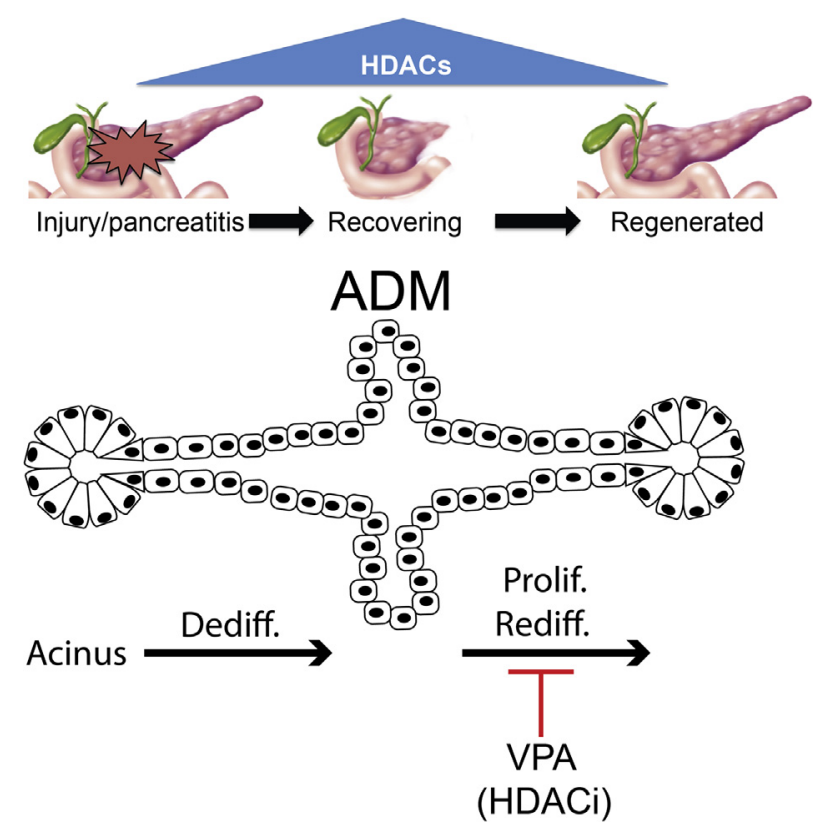

Figure 8 Model of histone deacetylase (HDAC) regulation during pancreatic recovery after injury. HDAC expression increases after pancreatic injury with peak expression at day 3 coinciding with peak pancreatic injury. As the pancreas recovers, HDAC expression decreases. Valproic acid (VPA) inhibits pancreatic recovery, allowing acinar-toductal metaplasia (ADM) complexes to persist and reduces acinar cell proliferation. Dediff., dedifferentiation; HDACi, HDAC inhibitor; Prolif., proliferation; Rediff., redifferentiation. 
TCF4 from pancreatic tissue nuclear extracts, we found that $\beta$-catenin associated with TCF4 for a short period during early recovery (at day 1 after injury) and this association was lost by day 3 (Figure 7). VPA administration, however, caused $\beta$-catenin to bind to TCF4 for a longer period (at least until day 3) (Figure 7). The result is consistent with the function of HDACs in facilitating the removal of $\beta$-catenin from the TCF complex. ${ }^{47,48}$

\section{Discussion}

We found for the first time, to our knowledge, a mechanism by which VPA predisposes individuals to pancreatitisthrough the inhibition of HDACs, leading to i) reduced acinar cell proliferation, ii) the persistence of ADMs, and iii) an arrest in acinar redifferentiation (Figure 8). Consistent with previous work, ${ }^{9}$ VPA administration by itself failed to induce pancreatic injury; in the setting of injury, however, VPA delayed recovery. This is also the first demonstration of the practical application of a newly developed digital image analysis tool that uses machine-learning algorithms to reliably quantify acinar content from standard H\&E-stained pancreatic sections. ${ }^{3}$ Mice receiving VPA after pancreatitis induction had $40 \%$ to $50 \%$ reduced acinar content a week after pancreatic injury compared with mice receiving only caerulein and not VPA.

We believe the mechanism by which VPA delays pancreatic recovery is through the inhibition of HDACs. This is primarily because the non-HDACi analog of VPA failed to mimic the delay in recovery seen with VPA. Further evidence suggesting a role for HDACs in pancreatic recovery is that several of the HDACs, particularly HDAC1, were up-regulated during recovery. HDAC1 is a class 1 HDAC that is localized to the nucleus and functions to deacetylate lysine residues on histone tails. We cannot exclude, however, the importance of nonnuclear HDACs in modulating pancreatic recovery through the posttranslational regulation of nonhistone proteins. ${ }^{49}$ VPA, however, primarily targets HDAC1 and other nuclear HDACs, and it does not appear to affect cytoplasmic HDACs. ${ }^{50}$

The higher HDAC expression we observed during pancreatic recovery is similar to what was reported during pancreatic development by Haumaitre et al. ${ }^{16}$ They also found that HDACi shifted the expansion of pancreatic precursors in a rat embryonic explant from an acinar to $\beta$-cell phenotype, suggesting that HDACs play a role in the maturation of the embryonic precursors into acinar cells. ${ }^{17}$ It has been known for some time that during pancreatic recovery, some of the acinar cells transiently assume characteristics of embryonic precursors. ${ }^{4,18,19}$ They dedifferentiate into tubular structures also known as ADMs that co-express both acinar and ductal proteins and undergo activation of several, embryonic-dominant signaling pathways, including hedgehog, notch, and wnt $/ \beta$-catenin. The mechanism by which HDACs modulate pancreatic regeneration in this context appears to be through promoting the redifferentiation of ADMs into acinar cells. Among signals, temporal $\beta$-catenin nuclear translocation and activation are necessary to induce ADM formation. However, its continued activation prevents redifferentiation and hence is associated with persistence of ADMs. ${ }^{20,51}$ We found that VPA prolonged the association of $\beta$-catenin to its nuclear complex or, in other words, delayed the temporal repression of $\beta$-catenin normally achieved by the HDACs. The repression is driven by HDAC-mediated deacetylation of histone tails located near $\beta$-catenin target genes. ${ }^{47,48}$

In summary, we provide new evidence that VPA use predisposes to pancreatic injury by inhibiting pancreatic HDACs and provoking an imbalance in pancreatic recovery. VPA appears to impede the redifferentiation of ADMs into mature acinar cells. The findings implicate HDACs as an important epigenetic switch required for the regenerative programs to properly reach completion.

\section{Supplemental Data}

Supplemental material for this article can be found at http://dx.doi.org/10.1016/j.ajpath.2015.08.006.

\section{References}

1. Stanger BZ, Hebrok M: Control of cell identity in pancreas development and regeneration. Gastroenterology 2013, 144:1170-1179

2. Murtaugh LC, Keefe MD: Regeneration and repair of the exocrine pancreas. Annu Rev Physiol 2015, 77:229-249

3. Eisses JF, Davis AW, Tosun AB, Dionise ZR, Chen C, Ozolek JA, Rohde GK, Husain SZ: A computer-based automated algorithm for assessing acinar cell loss after experimental pancreatitis. PLoS One 2014, 9:e110220

4. Jensen JN, Cameron E, Garay MV, Starkey TW, Gianani R, Jensen J: Recapitulation of elements of embryonic development in adult mouse pancreatic regeneration. Gastroenterology 2005, 128:728-741

5. Peery AF, Dellon ES, Lund J, Crockett SD, McGowan CE, Bulsiewicz WJ, Gangarosa LM, Thiny MT, Stizenberg K, Morgan DR, Ringel Y, Kim HP, Dibonaventura MD, Carroll CF, Allen JK, Cook SF, Sandler RS, Kappelman MD, Shaheen NJ: Burden of gastrointestinal disease in the United States: 2012 update. Gastroenterology 2012, 143:1179-1187.e1-3

6. Wu BU, Conwell DL: Acute pancreatitis part I: approach to early management. Clin Gastroenterol Hepatol 2010, 8:410-416. quiz e56-8

7. Forsmark CE, Baillie J: AGA Institute technical review on acute pancreatitis. Gastroenterology 2007, 132:2022-2044

8. Gerstner T, Busing D, Bell N, Longin E, Kasper JM, Klostermann W, Hebing B, Hanefeld F, Eckel U, Hoffmann R, Bettendorf U, Weidner B, Wiemer-Kruel A, Brockmann K, Neumann FW, Sandrieser T, Wolff M, Konig S: Valproic acid-induced pancreatitis: 16 new cases and a review of the literature. J Gastroenterol 2007, 42: $39-48$

9. Walker RM, Smith GS, Barsoum NJ, Macallum GE: Preclinical toxicology of the anticonvulsant calcium valproate. Toxicology 1990 , $63: 137-155$ 
10. Monti B, Polazzi E, Contestabile A: Biochemical, molecular and epigenetic mechanisms of valproic acid neuroprotection. Curr Mol Pharmacol 2009, 2:95-109

11. Nalivaeva NN, Belyaev ND, Turner AJ: Sodium valproate: an old drug with new roles. Trends Pharmacol Sci 2009, 30: 509-514

12. Dokmanovic M, Clarke C, Marks PA: Histone deacetylase inhibitors: overview and perspectives. Mol Cancer Res 2007, 5:981-989

13. Johnstone RW, Newbold A, Matthews GM, Bots M, Cluse LA, Clarke CJ, Banks KM, Cullinane C, Bolden JE, Christiansen AJ, Dickins RA, Miccolo C, Chiocca S, Kral AM, Ozerova ND, Miller TA, Methot JL, Richon VM, Secrist JP, Minucci S: Molecular and biological analysis of histone deacetylase inhibitors with diverse specificities. Mol Cancer Ther 2013, 12: 2709-2721

14. Minucci S, Pelicci PG: Histone deacetylase inhibitors and the promise of epigenetic (and more) treatments for cancer. Nat Rev Cancer 2006, 6:38-51

15. Ververis K, Hiong A, Karagiannis TC, Licciardi PV: Histone deacetylase inhibitors (HDACIs): multitargeted anticancer agents. Biologics 2013, 7:47-60

16. Haumaitre C, Lenoir O, Scharfmann R: Histone deacetylase inhibitors modify pancreatic cell fate determination and amplify endocrine progenitors. Mol Cell Biol 2008, 28:6373-6383

17. Haumaitre C, Lenoir O, Scharfmann R: Directing cell differentiation with small-molecule histone deacetylase inhibitors: the example of promoting pancreatic endocrine cells. Cell Cycle 2009, 8:536-544

18. Siveke JT, Lubeseder-Martellato C, Lee M, Mazur PK, Nakhai H, Radtke F, Schmid RM: Notch signaling is required for exocrine regeneration after acute pancreatitis. Gastroenterology 2008, 134: $544-555$

19. Fendrich V, Esni F, Garay MV, Feldmann G, Habbe N, Jensen JN, Dor Y, Stoffers D, Jensen J, Leach SD, Maitra A: Hedgehog signaling is required for effective regeneration of exocrine pancreas. Gastroenterology 2008, 135:621-631

20. Morris JP 4th, Cano DA, Sekine S, Wang SC, Hebrok M: Betacatenin blocks Kras-dependent reprogramming of acini into pancreatic cancer precursor lesions in mice. J Clin Invest 2010, 120:508-520

21. Winkler I, Blotnik S, Shimshoni J, Yagen B, Devor M, Bialer M: Efficacy of antiepileptic isomers of valproic acid and valpromide in a rat model of neuropathic pain. Br J Pharmacol 2005, 146: 198-208

22. Dowdell KC, Pesnicak L, Hoffmann V, Steadman K, Remaley AT, Cohen JI, Straus SE, Rao VK: Valproic acid (VPA), a histone deacetylase (HDAC) inhibitor, diminishes lymphoproliferation in the Fas -deficient MRL/lpr(-/-) murine model of autoimmune lymphoproliferative syndrome (ALPS). Exp Hematol 2009, 37: 487-494

23. Wildi S, Kleeff J, Mayerle J, Zimmermann A, Bottinger EP, Wakefield L, Buchler MW, Friess H, Korc M: Suppression of transforming growth factor beta signalling aborts caerulein induced pancreatitis and eliminates restricted stimulation at high caerulein concentrations. Gut 2007, 56:685-692

24. Strobel O, Dor Y, Alsina J, Stirman A, Lauwers G, Trainor A, Castillo CF, Warshaw AL, Thayer SP: In vivo lineage tracing defines the role of acinar-to-ductal transdifferentiation in inflammatory ductal metaplasia. Gastroenterology 2007, 133:1999-2009

25. Bockman DE: Morphology of the exocrine pancreas related to pancreatitis. Microsc Res Tech 1997, 37:509-519

26. Bockman DE, Guo J, Buchler P, Muller MW, Bergmann F, Friess H: Origin and development of the precursor lesions in experimental pancreatic cancer in rats. Lab Invest 2003, 83: $853-859$

27. Criscimanna A, Speicher JA, Houshmand G, Shiota C, Prasadan K, Ji B, Logsdon CD, Gittes GK, Esni F: Duct cells contribute to regeneration of endocrine and acinar cells following pancreatic damage in adult mice. Gastroenterology 2011, 141: $1451-1462 . e 6$

28. Adler G, Hahn C, Kern HF, Rao KN: Cerulein-induced pancreatitis in rats: increased lysosomal enzyme activity and autophagocytosis. Digestion 1985, 32:10-18

29. Lerch MM, Gorelick FS: Models of acute and chronic pancreatitis. Gastroenterology 2013, 144:1180-1193

30. Saluja A, Saito I, Saluja M, Houlihan MJ, Powers RE, Meldolesi J, Steer M: In vivo rat pancreatic acinar cell function during supramaximal stimulation with caerulein. Am J Physiol 1985, 249: G702-G710

31. Yamaguchi H, Kimura T, Mimura K, Nawata H: Activation of proteases in cerulein-induced pancreatitis. Pancreas 1989, 4: $565-571$

32. Anastasi A, Erspamer V, Endean R: Isolation and structure of caerulein, an active decapeptide from the skin of Hyla caerulea. Experientia 1967, 23:699-700

33. Anastasi A, Erspamer V, Endean R: Isolation and amino acid sequence of caerulein, the active decapeptide of the skin of hyla caerulea. Arch Biochem Biophys 1968, 125:57-68

34. Eyal S, Yagen B, Shimshoni J, Bialer M: Histone deacetylases inhibition and tumor cells cytotoxicity by CNS-active VPA constitutional isomers and derivatives. Biochem Pharmacol 2005, 69: $1501-1508$

35. Wasserman M, Yagen B, Blotnik S, Papo N, Bialer M: Stereoselective pharmacokinetic analysis and antiepileptic activity of N-2-hydroxypropyl valpromide, a central nervous system-active chiral valproylamide. Ther Drug Monit 2001, 23:414-420

36. Kopp JL, Dubois CL, Hao E, Thorel F, Herrera PL, Sander M: Progenitor cell domains in the developing and adult pancreas. Cell Cycle 2011, 10:1921-1927

37. Kopp JL, Dubois CL, Schaffer AE, Hao E, Shih HP, Seymour PA, Ma J, Sander M: Sox9+ ductal cells are multipotent progenitors throughout development but do not produce new endocrine cells in the normal or injured adult pancreas. Development 2011, 138: 653-665

38. Kopp JL, von Figura G, Mayes E, Liu FF, Dubois CL, Morris JP 4th, Pan FC, Akiyama H, Wright CV, Jensen $\mathrm{K}$, Hebrok M, Sander M: Identification of Sox9-dependent acinar-toductal reprogramming as the principal mechanism for initiation of pancreatic ductal adenocarcinoma. Cancer Cell 2012, 22: $737-750$

39. Prevot PP, Simion A, Grimont A, Colletti M, Khalaileh A, Van den Steen G, Sempoux C, Xu X, Roelants V, Hald J, Bertrand L, Heimberg H, Konieczny SF, Dor Y, Lemaigre FP, Jacquemin P: Role of the ductal transcription factors HNF6 and Sox9 in pancreatic acinar-to-ductal metaplasia. Gut 2012, 61: $1723-1732$

40. Lerch MM, Lutz MP, Weidenbach H, Muller-Pillasch F, Gress TM, Leser J, Adler G: Dissociation and reassembly of adherens junctions during experimental acute pancreatitis. Gastroenterology 1997, 113: $1355-1366$

41. Fukuda A, Chiba T: Sox9-dependent acinar-to-ductal reprogramming is critical for pancreatic intraepithelial neoplasia formation. Gastroenterology 2013, 145:904-907

42. MacDonald BT, Tamai $\mathrm{K}$, He X: Wnt/beta-catenin signaling: components, mechanisms, and diseases. Dev Cell 2009, 17: $9-26$

43. Keefe MD, Wang H, De La OJ, Khan A, Firpo MA, Murtaugh LC: beta-catenin is selectively required for the expansion and regeneration of mature pancreatic acinar cells in mice. Dis Model Mech 2012, 5: 503-514

44. Murtaugh LC, Law AC, Dor Y, Melton DA: Beta-catenin is essential for pancreatic acinar but not islet development. Development 2005, 132:4663-4674 
45. Clevers H: Wnt/beta-catenin signaling in development and disease. Cell 2006, 127:469-480

46. Clevers H, Nusse R: Wnt/beta-catenin signaling and disease. Cell 2012, 149:1192-1205

47. Billin AN, Thirlwell H, Ayer DE: Beta-catenin-histone deacetylase interactions regulate the transition of LEF1 from a transcriptional repressor to an activator. Mol Cell Biol 2000, 20:6882-6890

48. Daniels DL, Weis WI: Beta-catenin directly displaces Groucho/TLE repressors from Tcf/Lef in Wnt-mediated transcription activation. Nat Struct Mol Biol 2005, 12:364-371
49. Moser MA, Hagelkruys A, Seiser C: Transcription and beyond: the role of mammalian class I lysine deacetylases. Chromosoma 2014, 123:67-78

50. Cunliffe VT: Eloquent silence: developmental functions of Class I histone deacetylases. Curr Opin Genet Dev 2008, 18: 404-410

51. Zhang Y, Morris JP 4th, Yan W, Schofield HK, Gurney A, Simeone DM, Millar SE, Hoey T, Hebrok M, Pasca di Magliano M: Canonical wnt signaling is required for pancreatic carcinogenesis. Cancer Res 2013, 73:4909-4922 